\title{
Binding Significance to Form: Cultural Objects, Neural Binding, and Cultural Change $e^{\sqrt[3]{4}, \dagger}$
}

\author{
Marshall A. Taylor, Dustin S. Stoltz, and Terence E. McDonnell \\ Department of Sociology, University of Notre Dame
}

\begin{abstract}
In sociology, a cultural object is the "binding" of significance to a material form. But, how do people "bind" otherwise discrete elements as a single element? In cognitive neuroscience and the philosophy of mind, this is known as the "binding problem." Sociologists can learn from research on binding, as it deepens our understanding of cultural objects, learning, and social change. Binding is the process by which a material "token" is assimilated into (or expands the boundaries of) a cognitive "type," or resists such typification thereby leading to the formation of a new cognitive "type." Cultural objects are simultaneously types and tokens, and the interplay between them results in a fundamental cultural instability. Binding is an attempt to stabilize meaning in two ways: the first, innovating, is implicated in the emergence of a cultural object, and the second, indexicalizing, in its maintenance and extension. However, even the process of indexicalizing a well-established type -i.e., the proliferation of tokensprovides the material fodder from which to innovate new types. Attention to binding processes in the production and reception of cultural objects reveals important insight into the dynamics of cultural change and stability.
\end{abstract}

\footnotetext{
A version of this paper was presented at the 2018 Annual Meeting of the American Sociological Association in Philadelphia, and at the 2017 Mini-Conference on Culture and Cognition at the Eastern Sociological Society Meeting in Philadelphia. Direct all correspondence to Marshall A. Taylor, Jenkins-Nanovic Hall 4046, Department of Sociology, University of Notre Dame, Notre Dame, IN, 46556; e-mail: mtaylo15@nd.edu.

${ }^{\dagger}$ This paper is published in Poetics: Journal of Empirical Research on Culture, the Media and the Arts. The published version can be found here: https : //www.sciencedirect. com/science/article/pii/S0304422X18300275.
} 


\section{Introduction}

Thirty years ago, Griswold argued a sociology that takes meaning seriously "begins by focusing cultural analysis on the point at which individuals interact with a cultural object" (Griswold 1987a:4). But what is a cultural object? Griswold offers a succinct definition: "shared significance embodied in form" (Griswold 1986:5, 1987a:4, 2012:11). Cultural objects are composed of meaning and material, and more specifically, the binding of significance to a material form ${ }^{1}$ People experience material objects directly via perception (audibly, visually, tactilely, proprioceptively, or via smell and taste) or indirectly through conceptual information (e.g., conversation, diagrams, or texts). As Griswold (1987b:1080) argued, "when a cultural object engages at least some of the presuppositions of the person encountering it, meaning is "fabricated." It is this complement-these presuppositions in longterm memory and the material objects giving rise to or otherwise activating them-that we investigate here.

This leads to our main question: how are discrete elements "bound" into a single element by the brain? This is referred to as the "binding problem" in cognitive neuroscience and the philosophy of mind. "Binding" entails processes through which separate chunks of discrete information-being processed at different times, places, or with different modalities and neurocognitive structures - become associated in a non-random fashion creating new or sustained phenomenological experiences (see Whitney 2009:R251). While objects and situations could hypothetically divide into an indefinite number of units (devolving into an infinite regress), the mind effortlessly associatesbindsqualities of our experience into meaningful wholes.

We import research on binding to better understand the tension between what Patterson (2014) conceptualizes as meaning making and meaning maintenance. We argue processes of meaning-making and maintenance are not only grounded in distinct cognitive processes, but also maintenance is impossible without incremental moments of meaning-making. Certainly, this process of meaning-making and maintenance is informed by social processes (i.e., interaction, diffusion, exchange, structural inequalities) encouraging stable consensus or upsetting the status quo. However, for brevitys sake, we bracket many of these social processes to privilege the theorization of these binding processes, noting how this opens up the process to future inquiries.

\footnotetext{
${ }^{1}$ When we say form, we mean material form.
} 
We consider two ways binding processes shape cultural objects. The first, innovating, is implicated in the emergence of the cultural object. While people do not typically invent and reinvent meaning when making or confronting an object, they sometimes do. For sociologists, then, understanding innovating helps account for the process of making cultural objects - binding together new types encompasses the experience of new tokens. The second, indexicalizing, engages the maintenance and extension of cultural objects. When people impose meanings on an object, those objects can resist, forcing people to expand, contract, or otherwise revise those schematic networks comprising that type. In this way we account for how material form encourages revision of cognitive types.

We demonstrate how bridging research on binding and cultural objects is consequential to the practice of cultural sociology. Our working model of cultural objects is deficient without understanding these different paths to meaning; innovating and indexicalizing require different cognitive processes and different kinds of "cultural work" to stabilize (Edgell Becker 1998; McDonnell 2016). Furthermore, and significantly for cultural sociology, we find the kernels of cultural change are always entwined in the very processes producing and maintaining cultural objects.

In what follows, we first define what is meant by "cultural object," specifically distinguishing between two analytical aspects of cultural objects - types and tokens - that, while necessarily complementary and reciprocal, are analytically orthogonal to one another and are therefore each implicated in different ways in the production of cultural objects. By types, we refer to the result of cognitive processes of clustering aspects of experience. By tokens, we refer to material objects experienced as discrete entities, which may instantiate a type or resist a type (thereby creating new types). Considering cultural objects as types and tokens, and the relationship between the two, leads us to consider how human cognitive capacities - memory and perception, specifically - are crucial for explaining how cultural change happens by way of attempts at everyday cultural production and maintenance. We introduce the "binding" concept to theorize how cultural objects as tokens become bound to cultural objects as types (and vice versa). We then detail our two binding processes with a variety of empirical examples from contemporary Western art. 


\section{The Study of Cultural Objects meets the Study of Culture and Cognition}

To challenge the notion that people are perpetually in a (cognitively exhausting) state of meaning-making, Patterson (2014:8) provocatively asks, "when last did you make a meaning?" Most meaning, he argues, is already implicitly agreed upon, a product of an interactionally sustained cultural order. It follows that most objects are cultural objects in that we share agreement about what an object "is," what it "means" to us, and what we can "do" with it. If meaning-making cannot follow the "cognitively exhausting manner proposed by many conventional cultural sociologists" (2014:8), what form can it take? Patterson is surely right (drawing on Heise [2002]) that people are primarily meaning maintainers. That said, we worry this orientation (1) dismisses meaning making too hastily and (2) treats the meanings that people maintained as too settled. The binding problem usefully overturns conventional approaches to meaning-making as it relates to the study of cultural objects. We suggest meaning-making and meaning-maintenance are two distinct, but interacting, meaning processes grounded in binding significance to form. Before proceeding, however, we first must consider the question: what is a cultural object?

\subsection{Cultural Objects as Types and Tokens}

A wide range of examples of cultural objects are put forward in extant sociological literature. Griswold includes the following: sentences, epics, a YouTube video, bread, novels, plays, pots, the Black Hills, genres, shawls, martial arts, cigarettes, carved marble, folktales, an opera, gardens, canals, city comedy, a quilt, a sonnet, hairstyles, the Balinese cockfight, and a religious doctrine (to name a few). Sociologists building on Griswold additionally offer, for example: theater (Berezin 1994), prime-time television (Bielby and Bielby 1994), the Eucharist (Edgell Becker 1999), and the heirloom tomato (Jordan 2007).

How can we make sense of such variety? Griswold unpacks her definition further: "an expression of social meaning that is tangible or can be put into words" (1987a:4). Cultural objects are something we either sense or say. Implicit in this is the distinction between cultural objects as tokens and cultural objects as types (Peirce 1992). Griswold offers one particularly straightforward elaboration of this distinction: "a single play or a dramatic genre" (see Figure 11). Here, a "dramatic genre" is a type of which "a single 
play" may be a token. Not only are both types and tokens cultural objects, but types require tokens and vice versa. Cultural objects have an inherent "bipolar" structure mapping types to tokens (cf. Lizardo 2016). Tokens take an external form, and are always tangible and perceptible through the senses. Tokens, therefore, are necessarily physically instantiated, are not "ideational," and have qualities which exist independently of an individuals perception. Types, however, are mental, learned, and instantiated as cognitive processes. Therefore, cultural objects as types are mostly "put into words," or we refer to previously or currently experienced tokens to describe types, yet they are never exhausted by such instantiations.

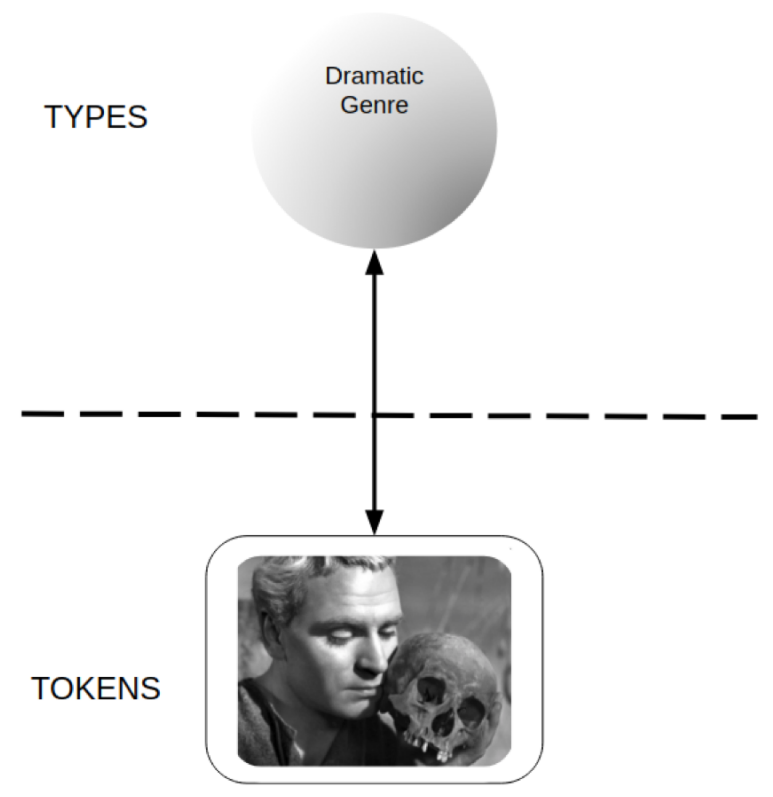

Figure 1: Cultural Objects as Types and Tokens

Note: Image from Laurence Olivier's Hamlet (1948).

The play-genre example is a clear demonstration of the relationship between tokens and types; however, there are two more complicating cases. The first is when a token becomes the exemplar of its own type. For example, the Statue of Liberty is a token of the type sculpture or public memorial; however, as there are many physical representations of the Statue of Liberty (e.g., souvenirs, pictures, etc.), this sculpture (as a tangible token) has become a type of which the actual statue in New York City is the exemplar. 
A second distinct case emerges when we consider even more abstract types (such as nationalism) in which a wide range of (phenomenologically) distinct tokens (flags, anthems, ceremonies, memorials) seem to be unified by their underlying type of shared significance.

In this latter case, intuition may lead some theorists of cultural objects to posit these sorts of abstract or intangible types are "immaterial" cultural objects. This, however, is misleading (McDonnell 2010) and often (implicitly or explicitly) invokes the Parsonsian-Geertzian imagery of a transcendental cultural system or seamless web "hanging above" social life. In contrast, we follow anthropologists (Hutchins 1995; Salomon 1997) and sociologists (Bourdieu 1977; Cerulo 2002) who argue "types" are distributed across the minds of those within particular communities - forming what Griswold refers to as the "horizon of expectations" (1987a:10). This shared reality carves into our bodies - literally transforming our neural architecture in material ways (Bloch 2015). These shared impressions are neither incommensurably fragmented nor evenly apportioned across individuals (Reay 2010). Therefore, there is never a single, all encompassing, pure or essential "type." Say we try to aggregate individuals' types of a particular cultural object for those in a relevant community. While we would find each description overlaps, there is never a pure, nor a whole description (see also Latour et al. 2012). Additionally, all types are built up and maintained only through iteration and routine engagement with tokensin other words, we "use it or lose it" (Martin 2010:232). Sociologists can benefit from recent developments in the cognitive sciences accounting for cognitive regularities involved in producing such types.

\section{Cognitive Regularities, Limitations, and Efficiencies}

Cultural sociologists must reconsider our implicit models of how brains produce and store types in long-term memory. Recently, sociologists confronting the brain's limitations have argued for the necessity of extra-individual phenomena as "scaffolding." Such cognitive limitations are of two (intertwined) varieties: processing (e.g., Biggart and Beamish 2003; Whitford 2002) and memory (Swidler 2001; Knorr-Cetina 2014).$^{2}$ We suggest com-

\footnotetext{
${ }^{2}$ In sociology, the evidence commonly cited to demonstrate the limitations of human memory relates to "working memory." This is not memory per se, however, as it only refers to the serial manipulation of contents during in-the-moment cognitive processing.
} 
plementing these accounts of cognitive limitations with consideration of cognitive regularities.

\subsection{Long-Term Memory and Neural Reuse}

Sociologists often (problematically) presume memory is an undifferentiated substance or a set of delimited and equal units (Lizardo 2017; Patterson 2014). This default model of (long-term) memory imagines experiences as more or less automatically imbibed and stored in the brain, like saving a video to a hard drive. This suggests cultural objects in the world are somehow placed into the mind, but remain equivalent to the objects in the world (see for example Parsons, Shils, and Smelser [1965] 2001:22). Although humans do have long-term autobiographical memory which approximates this notion (Tulving 2002), this is a small fraction of what we "know," and is not the default "format" for long-term memory.

Cognitive anthropology provides a better model of long-term memory. They first offered an early, but ultimately inadequate, alternative to the "video" model: the list of rules as in a decision tree or checklist (e.g., Goodenough 1956). Responding to this approach, Fillmore (1975) developed an influential alternative to the checklist theory of meaning in which he argued,

... a word like bachelor did more than merely designate an unmarried man; the word posited a simplified world in which (back in the day when people were more concerned about such matters) a man was expected to marry at a certain age, and bachelor designated those men who remained unmarried beyond that expected age. (Quinn 2011:35)

This motivated decades of anthropological research into the nature of "cultural models" (DAndrade and Strauss 1992; Holland and Quinn 1987; Shore 1996).

But how is a Fillmore's simplified world less cognitively demanding than a checklist? The solution is schemas: networks of differentially weighted neural pathways forming conceptual/perceptual information stored in long-term memory, activated during in situ cognitive processing. A difference with the common usage of this term in sociology, is that schemas are not "units," but rather recycled memory traces (Wood et al. 2018). These traces are themselves implicated as nodes between other networks, forming "multiple, nested, and overlapping neural coalitions, in which each neural element is a 
member of multiple different coalitions and cooperates with a different set of partners at different times" (Anderson 2016:1). To grasp how the human brain appears so limited and frugal, while simultaneously permitting the recognition and adaptation to highly variable environmental stimuli, this associational, reusable, promiscuous, and loosely "nested" organization of schemas is central (Martin 2010). We argue cultural objects as types are organized schematically, comprising loosely associated traces of past engagements with material tokens.

\subsection{Cognitive Processing and the Role of the Environment}

Just as sociologists' implicit models of memory are outdated, so too our understanding of cognitive processing. Complementary to the folk model of memory as a tape reel is the model of thinking as computer processing (D'Andrade 1987). This model conceives of the brain as a machine, computing "sequentially" on linguistic or mathematical code, directly referencing a metaphor of computer-based binary code (Cisek 1999). Furthermore, this model conceives of computing as isolated from the "real world." This traps us in pernicious epistemology, which John Searle refers to as "one of the biggest mistakes in philosophy in the past several centuries" (2015:10). Put simply, current sociological assumptions about cognitive processing over-privilege the role of rules or codes for meaning-making and maintenance at the expense of understanding how the production and upkeep of types are deeply shaped by patterns of embodied engagement with material tokens.

Conceptualizing "thinking" as a translation of perceptual experience into a private, symbolic language, makes it difficult (or even impossible) to account for "meaning" in a pragmatic sense. Referred to as the "symbolic grounding problem" (Lizardo 2016), Harnad puts the matter succinctly: "How can the meanings of the meaningless symbol tokens, manipulated solely on the basis of their (arbitrary) shapes, be grounded in anything but other meaningless symbols?" (Harnad 1990:335).

Parsimoniously, the alternative is the same approach above: cognitive processing is the reuse of sensorimotor-perceptual schemas in the formation of new neural "coalitions" (Barsalou 1999; Goldstone and Barsalou 1998). This model collapses the division between perception and cognitive processing, a key strength of the "embodied" approach to cognition (Ignatow 2007; Wilson 2002). Importantly, an individual's type is deeply connected with the process by which an individual experiences (and has experienced) the token (Glenberg 1996). The interpretation of cultural objects as tokens never 
reduces to a set of binary codes or exhaustive rules by which to derive typesbecause types are the schematic traces of accrued embodied engagement with and tangible manipulation of tokens.

We endorse an understanding of cognition that recognizes the necessity of the present, physical and social environment for cognitive processing (Glenberg 1996; Gibson 2014). Human thinking "extends" beyond the organismic boundaries of our skin. It suffuses not just other people through language, imitation, and "mirroring" neurons (Bloch 2015), but also the physical setting of which we are always a part. That cognition cannot be confined to an individual's brain incorporates various approaches such as "distributed cognition" (Hutchins 1995), the "extended mind" (Menary 2010), "situated cognition" (Clancey 1997), and "scaffolded" cognition (Lizardo and Strand 2010).

While the "meaning" of objects involves the reconstruction of schematic "simplified worlds" in the mind, the features of that simplified world rely on the structure and content of our present environment (Glenberg 1996). Our brains are tools for the navigation and manipulation of material objects in a physical world (Gibson 2014). Therefore, "we can use the world as its own best model and visit and revisit the real-world scene, sampling it in detail at specific locations as required" (Clark 1998:29). The physical, non-human world does not come to us as an undifferentiated mass waiting for us to impart order by imposing our (socially constructed) "grid of perception" (see Martin 2011 for an extended critique). Rather the physical world is already "pre-ordered" in a mundane sense, and "affordances" emerge through the situational interaction of our bodily/cognitive capacities and the qualities of materials in the moment (McDonnell 2010, 2016; Gibson 2014; Stoltz and Taylor 2017).

\subsection{The Relationship Between Types and Tokens: From Crisp Sets to Fuzzy Sets}

Some cultural theorists conceptualize a "unit" of culture as if it could be disembodied and immaterial. Commensurate with the metaphor of "internalization," as used by Parsons (1964), this suggests that units are taken from the outside world and placed inside the head of an individual, where they become available for the individual to use at a later time (either sincerely or cynically). In this model, there is a one-to-one correspondence between the public unit and the personal unit. The set of units in the world is equal to the set of units in the mind. This is dubbed the "fax" (D'Andrade and 
Strauss 1992:811) or "downloading" view (Vaisey and Lizardo 2016) of internalization, by which public culture is copied directly as mental analogues in the mind (Shore 1996:52).

In contrast, we suggest sociologists follow cognitive linguists, psychologists, and anthropologists by moving our conceptualization of culture and enculturation from a model of crisp sets to fuzzy sets. Rather than assimilating tokens to types based on mutually exclusive and globally exhaustive criteria (i.e., crisp sets), types assimilate tokens following graded membership criteria (i.e., fuzzy sets) (Lakoff 1999; Rosch 1999) ${ }^{3}$ In a crisp set approach, a token is either a member of a type or it is not based on a list of criteria which must be met. In a fuzzy set approach, a token may have partial membership in multiple types and never perfectly instantiate any one type.

Take, for example, Italian artist Gianluca Gimini, who asked people to draw a bicycle completely from memory. Gimini "[s]oon ... found out that when confronted with this odd request most people have a very hard time remembering exactly how a bike is made. Some did get close, some actually nailed it perfectly, but most ended up drawing something that was pretty far off from a regular mens bicycle" (Kenny 2016). After collecting almost 400 sketches, Gimini created three-dimensional renderings (see Figure 2). The myriad engagements with a particular material token as people put them to use are "internalized" in the mind as a fuzzy set of attributes, schematic traces which, over time, coalesce into a more or less distinct "type." People who have only seen bicycles have a different schematic mapping of the attributes of "bike" compared to those who know how to ride a bike, compared to those who compete in the Tour de France. People who engage with token bicycles develop a capacity to identify "bicycle-like" tokens in the wild, discerning the difference between bicycle and tricycle at a minimum, or fixed gear versus free wheel for more discerning bike enthusiasts. This capacity can then "generate" new tokens, such as a drawing of a bicycle (see Figure 3). The same person, even if they tried very hard, would not generate the exact same drawing, but would instead produce slight variations with each

\footnotetext{
${ }^{3}$ Scholars of categorization or typification following this tradition commonly use "family resemblances" or "prototypicality" to refer to this same feature of types. For example, Lakoff and Johnson (1980:xi) specifically cite Wittgenstein and Rosch as well as the inventors of fuzzy set theory, Lotfi Zadeh and Joseph Goguen, as inspiring their approach to "image schemas."
} 

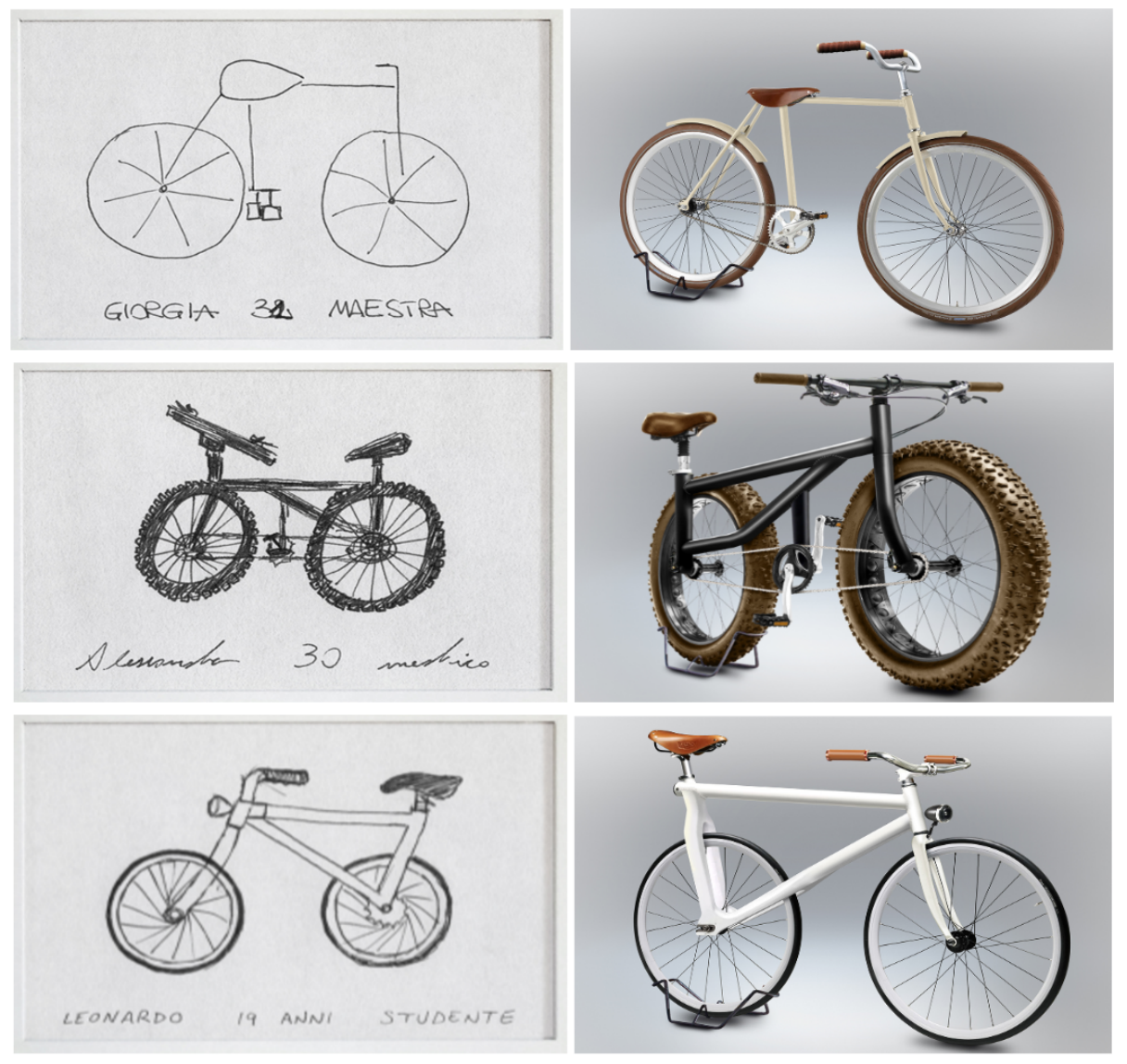

Figure 2: Gianluca Gimini, Velocipedia, 2009. Reproduced with artist's permission.

iteration $4^{4}$ People with different histories with bicycles would articulate and emphasize different qualities of bicycles, informed by their differential careers of engagement with bikes.

That everyday actors' types are very different from analysts' types is a key insight of the ethnomethodologists (Heritage 1987). Scholars strive for crisp sets in scholarly endeavors, but outside this sliver of attention and activity, they rely on fuzzy sets. Bolstering the ethnomethodologists' account

\footnotetext{
${ }^{4}$ See also Google's "Quick Draw" dataset, in which over 15 million people produced 50 million sketches over 345 categories. See https://github.com/googlecreativelab/ quickdraw-dataset
} 


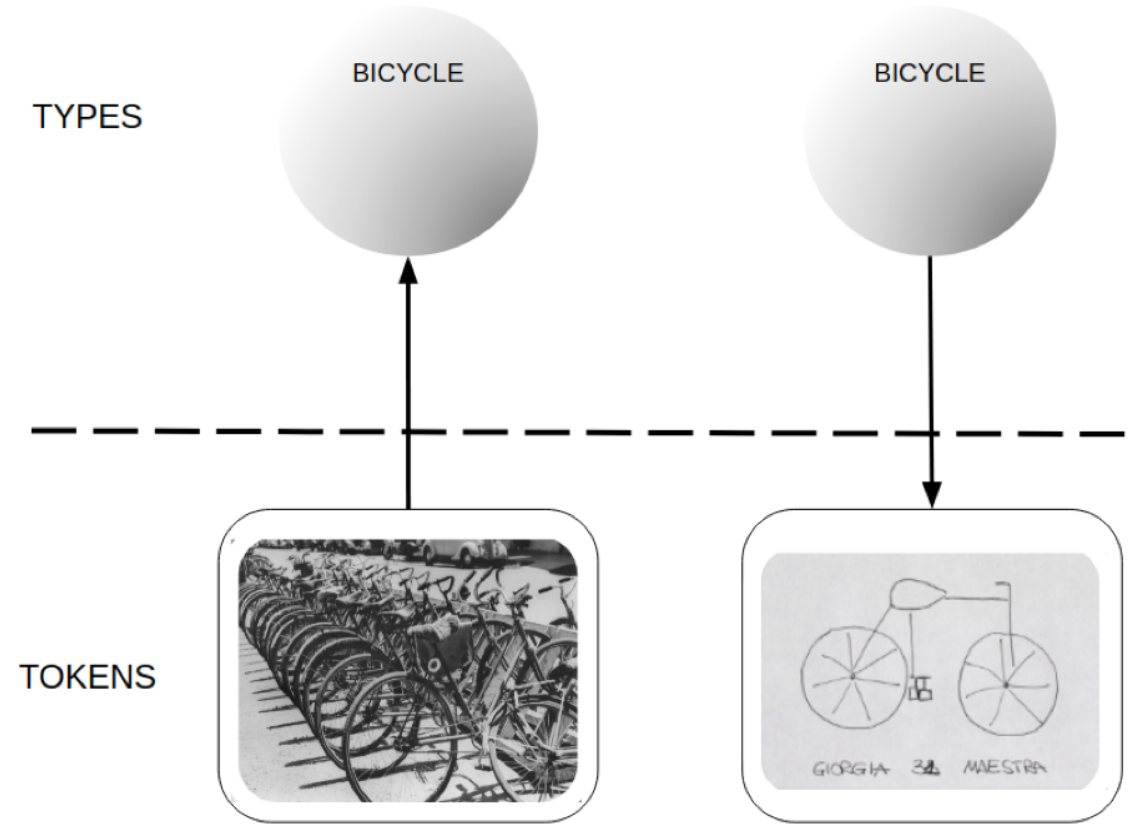

Figure 3: The Relationship Between Types and Tokens

is the importance of materiality in complementing our fuzzy types and distributing memories across time and space. On one hand, neural pathways are durable, yet always updating to accommodate discriminate features of an experience with a token. On the other hand, materials are durable, yet always malleable - being shaped by both human and non-human processes. In this way we require a vision for cultural objects grounded in revision.

\section{The Binding Problem: How Does Significance Get Stuck to Form?}

How do these insights alter how cultural sociologists understand cultural objects? Moving to a new understanding of how cognition works forces us to rethink how significance becomes tied to form. The old model saw cognition as organized around crisp sets (semiotic codes), storing objects whole cloth in 
memory as disembodied representations. It viewed people processing tokens out in the world through a checklist of rules. This model has a conservative bias that cannot effectively account for change. If what is shared is a faxed replication of objects and a checklist of qualities that make them in or out of a type, how do we account for interstitial cultural objects or variation among people in internalization? In other words, it does not give us a good account of how novel cultural object emerge, or come to resonate.

Rather people think through fuzzy types based on experience, carry incomplete memories of objects bolstered by material environment and social interaction, and process tokens in the world through neural associations (coalitions), often in non-linear ways. Given this understanding, the binding of significance to form is much more unstable than previously assumed.

For one, cultural objects (as tokens) are never completely internalized (as types) and people's perceptual capacities and personal histories mediates what is internalized. These "transcription errors" mean schematic associations are always partially idiosyncratic. More than just varying at the group level, meanings of cultural objects vary at the level of personal culture (Lizardo 2016). People's unique experiences with tokens in the world lead to different personal schematic associations. These associations imperfectly overlap with public codes. Perceptual variation and experience with the material world lead to different meanings in different interpretive arrangements (McDonnell 2016). People's personal experience interacting with tokens accumulates over time, leading to the emphasis of some qualities of a cultural object over others. In these ways, attention to binding processes makes visible the emergence of unexpected associations and meanings when interacting with objects. The implications of such individual level variation is often taken for granted in studies of reception and meaning. These formal dimensions of cognitive processing offer an understudied source of dynamism in the production and reception of cultural objects. Cultural objects (as types) are constantly being revised in the mind's of individuals, which feeds back into meaning-making and meaning-maintenance in public culture.

With this new model in mind, we argue the vast theoretical and empirical work on neural binding in cognitive neuropsychology offers a framework from which to tackle these theoretical problems surrounding cultural objects. Neural binding refers to the general processes by which percepts/concepts are tied together to form meaningful "wholes" in the mind. Neural binding researchers ask: How do separate bits of information, despite being processed with distinct neurocognitive structures, and experienced through different 
perceptual modalities, coalesce to form "coherent perceptual experience[s]" (Whitney 2009:R251)?

This leads us to two questions at the heart of this paper: 1) Under what circumstances is a material object experienced as if it is a token of a unique and distinct type of "cultural object," as opposed to an already familiar type of cultural object? 2) As a cultural object becomes more "available" through proliferation of material instantiationsfalling on a wide range of resemblance, from exact replica, to iconic, to purely symbolic (in Peirce's [1992] sense of the word) - how do our brain binds all these particular tokens into a singular type? As the preceding revealed, neither mind nor materiality are sufficient.

\subsection{Binding Tokens to Types and Types to Tokens}

The discussion up to this point has served to provide the necessary vocabulary and frame of thought to address the following question: How does significance bind to form to create a cultural object, and how and why does this binding change over time and space? Said differently, how do tokens bind to types, and types to tokens? Using this vocabulary (summarized in Table 1), we can begin to find answers in the neural binding literature of cognitive neuroscience.

How does neural binding - one of the oldest and most debated theoretical issues in cognitive neuroscience (Feldman 2013; Zimmer, Mecklinger, and Lindenberger 2006) ${ }^{5}$ - shed light on the creation and maintenance of cultural objects? We highlight two processes through which people attach meaning to objects, what we call "innovating" and "indexicalizing." Innovating, as summarized in Table 1, refers to the ways neural coalitions are formed into a single novel type by way of interaction with ambiguous tokens. Indexicalizing refers to the ways types are instantiated over time in various tokens. We outline each process below, also illustrating how the complexity of these bindings may vary depending on the embodied cultural capital that an actor has at their disposal in the sense-making setting. We illustrate our discussion using a variety of examples from contemporary Western art ${ }^{6}$

\footnotetext{
${ }^{5}$ This field is wide-ranging - including perennial concerns like the nature of consciousness and our sense of temporality - but for our purposes we focus on variable binding to illustrate our point. Variable binding refers to the processes by which "symbols are designated to represent entities" (Browne and Sun 1999:189 - here, "symbol" is used in a sense common to artificial intelligence researchers and is synonymous with "variable."

${ }^{6}$ Responses to the art works in our discussion are more readily available than accounts
} 


\begin{tabular}{|l|l|}
\hline \multicolumn{1}{|c|}{ Terms } & \multicolumn{1}{c|}{ Definitions } \\
\hline Schema & $\begin{array}{l}\text { Networks of differentially weighted neural pathways forming } \\
\text { conceptual/perceptual information stored in long-term mem- } \\
\text { ory. }\end{array}$ \\
\hline Type & $\begin{array}{l}\text { The result of cognitive processes clustering aspects of expe- } \\
\text { rience. The association between schematic traces of accrued } \\
\text { embodied exposure and tangible manipulation of tokens. }\end{array}$ \\
\hline Token & $\begin{array}{l}\text { Material/perceivable objects experienced as (more or less) } \\
\text { discrete entities. }\end{array}$ \\
\hline Crisp Set & $\begin{array}{l}\text { Tokens are considered either in or out of types based on mu- } \\
\text { tually exclusive and globally exhaustive criteria. }\end{array}$ \\
\hline Fuzzy Set & $\begin{array}{l}\text { Tokens are placed into, potentially numerous, types based on } \\
\text { partially overlapping criteria, which is never exhaustive. }\end{array}$ \\
\hline Innovation & The process by which tokens are clustered into a new type. \\
\hline Indexicalization & $\begin{array}{l}\text { The process by which a token is identified as an instance of a } \\
\text { type, produced so as to instantiate a type, or alters a type to } \\
\text { make room for that token. }\end{array}$ \\
\hline
\end{tabular}

Table 1: Core Terms and Definitions

\subsection{The Innovation Problem}

How can different sensory experiences coalesce into a new and discrete "form"? We call this the innovation problem. The idea that collections of individual "things" can be phenomenologically experienced as composing a novel "thing" not reducible to its component parts is not new in sociological theory.7 What earlier accounts lacked, however, is a model of cognition to undergird their (more or less implicit) theories that presage the ability to make the bindings in the first place. People bind together previously discrete elements of the sociomaterial world and proceed to interact with these new

behind the production of the pieces of art themselves. For illustration purposes, then, we treat the art as a given; i.e., our analytical "starting point" is the art itself, and we consider processes of innovation and indexicalization following peoples engagement with the art object.

${ }^{7}$ Blumer (1969:101), for instance, suggested that what all physical entities (e.g., desks, chairs), social beings, and more abstract ideas such as ethics and philosophies share is "object" status: they are matter "that can be indicated or referred to." Sociological answers to how things come to obtain object status have gone by different names, such as typification (Berger and Luckmann 2011) and materialization (Slater 2002). 
bindings as entities, as cultural objects, in and of themselves. Tracing the process of innovation, therefore, sheds light on how distinct cultural objects are (cognitively) created.

Research on neural binding models the underlying cognitive processes involved in "innovating." First, neurons "that fire together wire together" (Hebb 1949; Keysers and Perrett 2004). That is, the situated experience of multi-modal perceptual-sensorimotor patterns in the course of active engagement with situations reinforces those same perceptual-sensorimotor neural associations. This cognitive process likely undergirds most instances of "new" bindings for individuals (developmentally). However, it cannot account for the emergence of a historically novel type - which tends to be the sorts of cultural objects sociologists study. A second process that results in a new mental type, previously unknown in the community, is referred to as "convolving." In this neurocognitive process, two or more alreadyentrenched schemas bind to form a novel one (Thagard 2010; Thagard and Stewart 2011). Thagard (2010) describes this as the Aha! experience-i.e., the sudden feeling that one has gained a new understanding on a problem or ambiguous situation, also known as the "subjective experience of insight" (Bowden and Jung-Beeman 2003:730). In this process of sense-seeking, two or more schemas bind to form a novel association, then the "cognitive appraisal" and "physiological perception" are overlaid to assess the importance of the bind, and, finally, "the combined representation and the integrated cognitive/physiological emotional response [are combined] into a unified representation (pattern of neural activity) of the creative representation and its emotional value" (Thagard and Stewart 2011:454). At the core of this process is neural reuse (Anderson 2010), and the production of a new neural coalition.

Take, for instance, Sarah Sze's Untitled (1997) 8 , an installation comprised of everyday objects of innumerous diversity, with distinct perceptual patterns, architectural structure, immense scale, and incredible detail. Despite all of this complexity, it nonetheless combines into a single object that is significant and, importantly, experienced as a discrete object not decomposable into these perceptual patterns. People can innovate new types with

${ }^{8}$ An image of this piece can be found at the Tanya Bonakdar Gallery: http://www.tanyabonakdargallery.com/artists/sarah-sze/series-sculptureandinstallation_7/40. Note that images may be added to this photo album, so this URL may not link to the appropriate image at a later date if new pieces are added. 


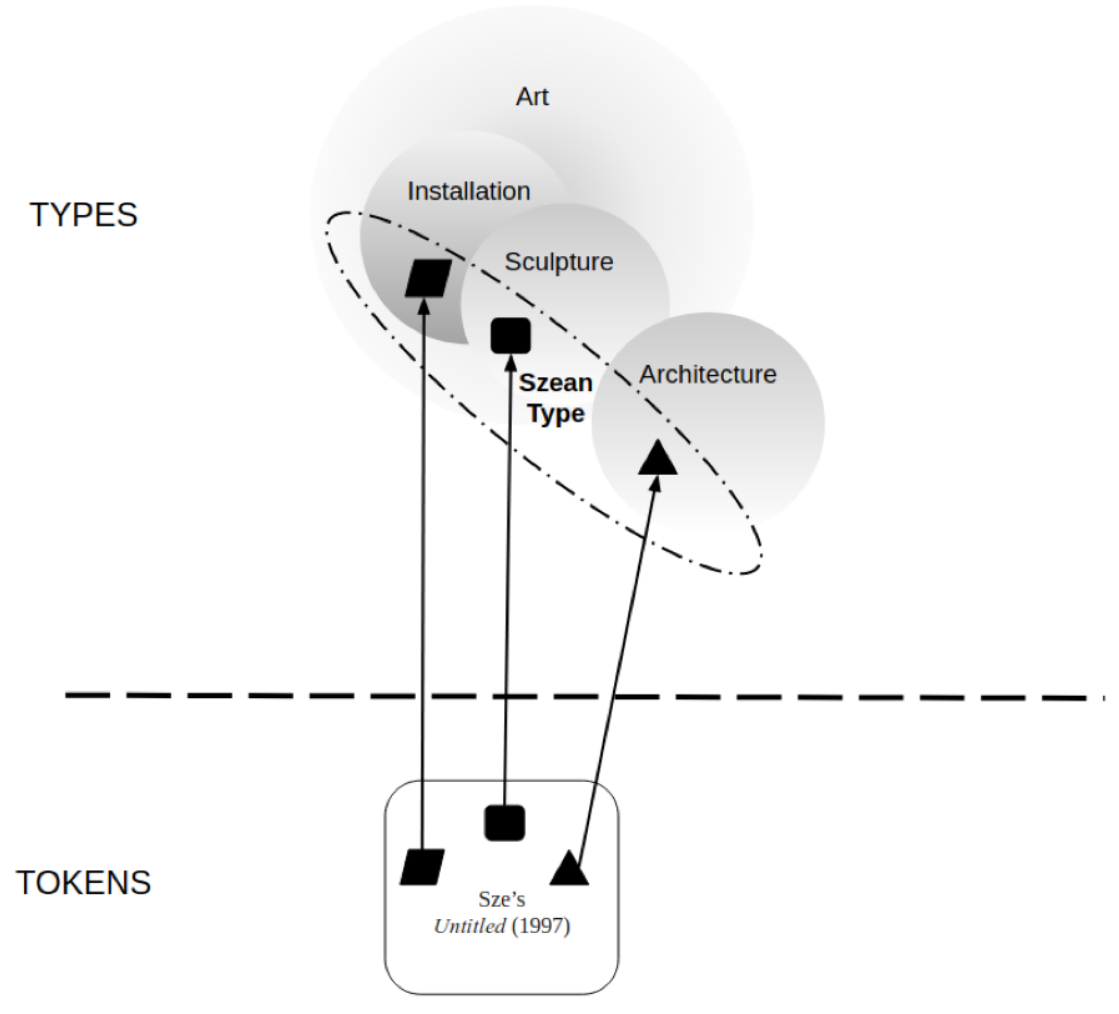

Figure 4: Innovating the Szean Type

Note: Black shapes portray qualities of the object in question.

Sze's work because the art sets up the opportunity for viewers to convolve schemas ranging from architecture and cities to sculpture, model building, everyday objects, and art installations into something altogether new (see Figure 4). Art critics struggle to cleanly categorize her work, describing it as "miniature landscape," "architecture," and "dollhouse" (Richard 2005); or about the relationships between "landscape, architecture, and sculpture and line" (Scott 2012). Critics remark on, and make manifest, the struggle to describe her work, referring to the "elusiveness" of Sze's work and defaulting into a listing the incongruous objects (Schwabsky 1999). For example:

The primary challenge of reviewing Sze is cataloguing her materi- 
als and their placement. To wit: The largest piece here consisted of an airy grid of orange and blue threads spread through the main space at an oblique angle to the floor, anchored between a column, wall, and a concatenation of what looked like toy ladders. Nestled under the implied plane of the grid like a city under a web of flight paths was the usual beguiling distribution of exactingly selected junk - a delta of red pushpins set upside down on their flat heads; a colony of small white gift boxes; a pair of jeans turned halfway inside out and balanced on their waistline, so that the upturned legs looked like twin cooling towers. Smears of red and blue pigment on the floor implied bloodstains and lagoons, "natural" touches that rhymed with a scatter of fake lichen and a live plant in a pot. (Richard 2005)

With objects resembling flight paths, a colony, twin cooling towers, lagoons, the metaphorical work here suggests how cognitively demanding Sze's work is.

Audiences meeting Sze's work may have trouble making sense of the work given pre-existing types. Interaction with a cultural object like Sze's artwork resists "type" such that a novel type is required to make sense of it, what we refer to as the "Sze type." In another essay, art critic Richard (2010) accounted for the Sze type by describing the "laboratory-playhouse-boutiquearchive of commodity that we expect from her" and even used the language of "Szean." When presuppositions fail to account for an object, people may create their own type by binding together attributes of previously held (perhaps more generic) types. This new, novel type made by audiences - the Sze type - is an innovation; an unfamiliar type that likely has some similarity to Sze's (the person's) type and to fellow audience members' types due to the material qualities of the object grounding perceptual experience.

Whether and how people innovate new types depends upon variation in people's presuppositions and perceptual capacities resulting from their particular life trajectories. It is here that distributions in embodied cultural capital can explain why a type may vary in complexity from one person to the next (Bourdieu [1979] 1984, 1993). As we've seen, art critics, and likely others with field-specific cultural capital around the arts, bind together different types based on artistic genre (e.g., landscape, sculpture, readymades), or resemblances to other artists (DuChamp, Whitman), into the Sze type. The contours of what is bound varies by the people's existing schematic avail- 


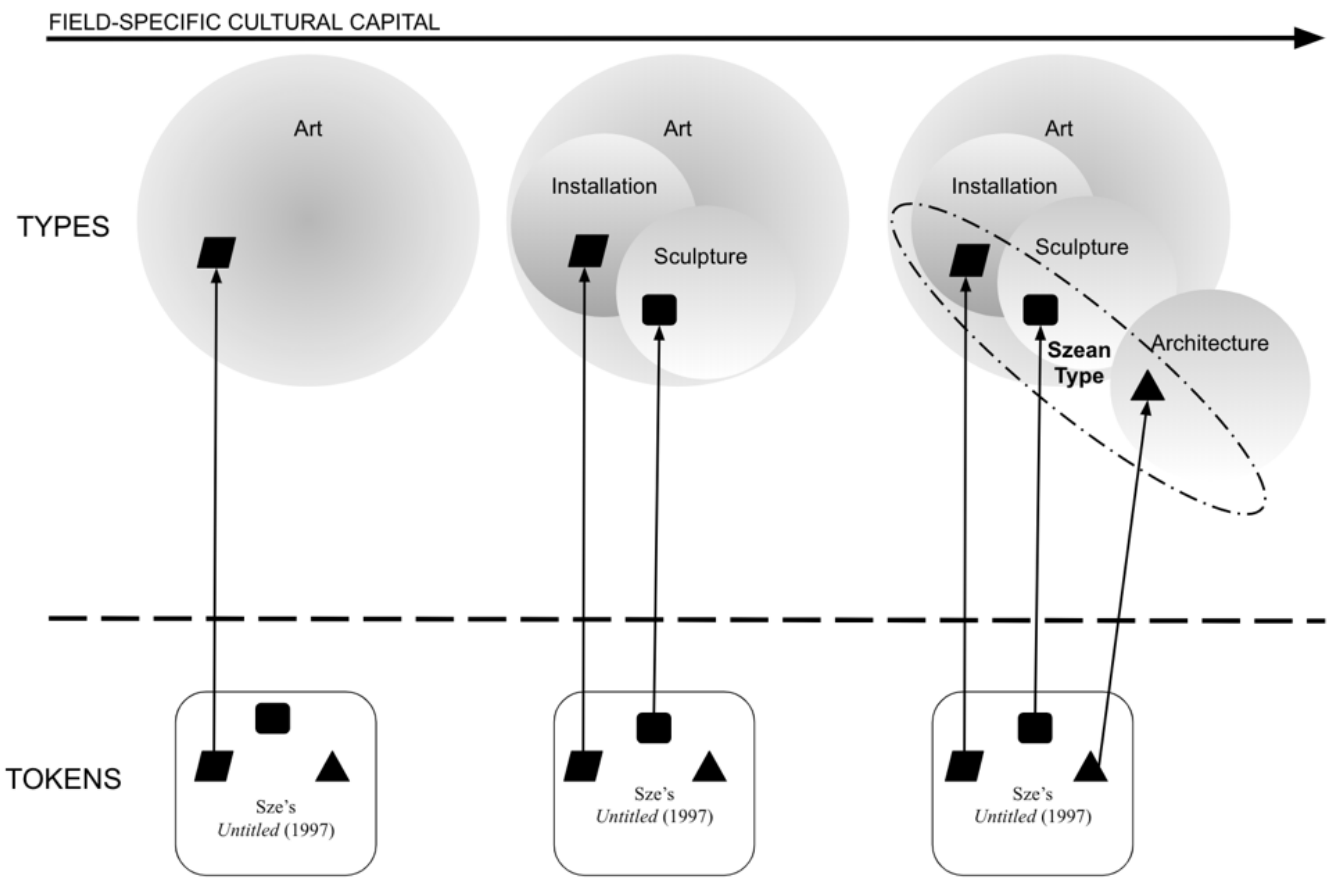

Figure 5: Cultural Capital and Innovation

Note: The "field-specific cultural capital" referenced in this figure is embodied cultural capital specific to the arts field. Black shapes portray qualities of the object in question.

ability (Tversky and Kahneman 1973) specific to the field in question and the actors' positions within it. Consider, for example, Figure 5. The panel farthest to the right repeats Figure 4, and illustrates how innovation might work for a person centrally positioned in the arts field (and who therefore is steeped in field-specific cultural capital relative to those positioned further out in the field). This person, by virtue of having "more" arts-related embodied cultural capital and engaging in arts-related practices more regularly, would be likely to innovate a more complex "Sze type" because they are in a better position to recognize (implicitly or explicitly) qualities of the particular Sze token that are cognitively associated with a more diffuse distribution of pre-existing art-related types (e.g., architecture, installation, and sculpture).

Someone closer to the center of Figure 5 - say, a city planner - may bind a Sze type differently, drawing on urban design schemes or seeing the work through the lens of, say, zoning. Of course, this is not to say that a city plan- 
ner lacks cultural capital in a sort of general, field-independent sense. Rather, we posit that the cultural capital relevant for a city planner to innovate a Sze type emerges from their longstanding interactions and problem solving in the field of urban infrastructure and development (assuming this city planner isn't simultaneously centrally positioned in a field more closely adjacent to the arts than municipality design). People with a narrow understanding of art as representationalist paintings and sculpture might find Sze's work too far afield from what they schematically understand as art and reject it out of hand - "that isnt art, and I dont care what it is." Still others with limited experiences at museums and galleries - say, those that would fall farther to the left side of Figure 7-might derisively call it "art," schematically classifying Sze's work as "objects found in museums I dont like or understand." In this way, as field-specific cultural capital is unevenly distributed, so is the capacity to innovate a type relevant to that field. Future ethnographic and interview-based research can test the extent to which this hypothesis holds water empirically, and may also help elucidate other dimensions of the innovating process that are mediated by field position (e.g., the amount of cognitive effort that goes into the binding, the emotional arousal of the binding, or the number of interactions and engagements with a particular token that are necessary for a new type to be created).

Once innovated, how are these novel cultural objectsas schematic "types" maintained across time and space? In other words, how are token representations of the same "type" created, and how are these tokens phenomenologically experienced as taking on the same "form" despite the fact that they may never be co-experienced in the same setting? Through iterations over time, Sarah Szes art as a type will be codified through experience (e.g., repeated engagements with her art objects) and circulated discourse (e.g., art criticism) around the type. In this way, clearer boundaries emerge around the type and shared significance is more firmly established. Once instantiated in an object, that type may "diffuse" by way of the further production of tokens of this novel type. This leads to a different kind of problem - that of type maintenance.

\subsection{The Indexicalization Problem}

If innovating is the process of producing "difference," we also need to theorize the production of "sameness." We call this the indexicalization problem. Indexicalizing is the process through which people take distinct tokens, which may be seldom or never co-experienced, to be of the same 
type.$^{9}$ Here we generalize from the notion of "indexical expressions" in ethnomethodology (Emirbayer and Maynard 2011; Garfinkel 1967:45; Giddens 2013:42) — wherein words and utterances are tokens of a more general and unverbalized intended meaning. Indexicalization ${ }^{10}$ refers to how discrete tokens come to be associated with one another and perceived to be (loosely or tightly) held together by the (largely unconscious) network of conceptual similarities they share and mutually activate when they are perceived. This occurs in the production or reception of new tokens as a particular type: e.g., the performances, year after year, around the globe of Shakespeare's Julius Caesar. In this way, indexicalization is important for cultural sociologists interested in the reproduction, maintenance, and revision of types through tokens.

While it is the case that maintaining the same token-also referred to as stabilization (Dominguez-Rubio 2014; Griswold, et al. 2013) - is not a static process, the key problem is how people fit pre-existing tokens to pre-existing types, or attempt to craft an object that fits a pre-existing type. Atypical qualities may call into doubt the schematic fit, activating the revision of the type, the filtering of those aspects of the object that don't match up, or a search for other types that might better match. Indeed, the brain is always undergoing this process in an effort to adequately make predictions about the world (Vatansever, Menon, and Stamatakis 2017). This means, however, that cultural change unavoidably seeps into even the most faithful of cultural reproductions.

A conception of culture as composed of crisp sets cannot handle such widespread mismatch between a type and the collection of material tokens that instantiate it. What tethers together all potentially discrete tokens is their fuzzy membership across types, including their partial fit in nested

\footnotetext{
${ }^{9}$ While there are obvious affinities between our argument and Berger and Luckmann's notion of typification as a process, there is an important difference. Specifically, we emphasize the necessity of materiality. We would underscore the grounded, physical, and experiential side of typification, rather than the abstract or ideal. Berger and Luckmann suggest that "[t]he social reality of everyday life is ... apprehended in a continuum of typifications, which are progressively anonymous as they are removed from the "here and now' of the face to face situation" (1966:33). We'd push back against the notion of typifications as "highly anonymous abstractions" and argue that cultural objects aren't just typifications, but material tokens as well.

${ }^{10}$ Our use differs slightly from Peirce's use of "index," in that the literature we draw upon uses the term "index" as roughly synonymous with Pierce's use of "sign."
} 
schematic coalitions (Jackendoff 2002; van der Velde and de Kamps 2006).11 In the language of this paper, these schematic coalitions are "types" which bind together perceptually similar and dissimilar "tokens." In this sense, a token need not have qualities that perfectly match the qualities of the type in order to indexicalize it; instead, it only needs enough of a family resemblance to other established tokens of the type. In such instances, the tokens are tethered together because they both mutually indexicalize the type - however imperfectly.

Of course, types can range from generic to specific. Very general types can tether perceptually diverse tokens. For example, our previous onlooker who simply sees Sze's work as "art" would likely see paintings, sculptures, and other installations as yet other indexicalization of the type "art." Generic types are important for cultural analysis, and certainly of social significance, but we presume that many cultural sociologists are more interested in specific types - such as the "Szean type" in Figures 6 and 7. In this case, when an historically novel binding emerges, it becomes indexicalized within a delimited social context so as to be meaningful to a particular community (e.g., Lena 2012:33-40).

Once innovated, bindings circulate, in part, through the production of tokens. But here, we must be careful not to fall into the trap of conceiving of the externalization-internalization relationships as one-to-one. Widely shared and entrenched types become familiar through broad circulation of object tokens, which increases opportunities for engaging with the object (Schudson 1989:160-4). Especially in the case of specific types, a community's iterative experience with the object(s) over time help affirm/reinforce the bindings within the minds of members of that community. For example, once Sze's style is established as a type for a particular community of art consumers, every new work she produces is then seen in light of that type-

\footnotetext{
${ }^{11}$ The literature we draw on refers to these workspaces, or "blackboard architectures," for the sake of clarity we do not introduce these terms. These are unconscious "workbenches" (Jackendoff 2002:200) that allow the relations between tokens to be mapped out. For example, as van der Velde and de Kamps (2006:43) illustrate, in making sense of the utterance "the little star is beside the big star," word symbols (for "little," "big," and "star") and grammatical relation symbols are able to "communicate" with one another by way of their connection to the working memory structure activated when the utterance was heard: in this way, the person can know that one star is little, the other star is big, and they are beside one another.
} 
which is, by definition, the process of indexicalizing. While each new token is read through this established type, it also potentially reshapes the type by introducing new (intended or unintended) qualities, or by making legible previously unacknowledged elements of the type - i.e., something that was always there, but never consciously realized and articulated.

While Sze's earliest work established the "Sze type" - that novel blend of sculpture, architecture, urban design, and everyday objects-her later work refines that type. As new tokens of Sze's work appear they point to the type, and audiences familiar with Sze's work then experience any new piece through that type. In turn, any new tokens affirm, revise, and sometimes demand dramatic rethinking of a type. As Sze's later work elevates off the ground, sometimes defying gravity, her work appears less grounded and urban, and more futuristic ((see Sarah Sze's Hidden Relief [2001] $]^{12}$ ). Imaginings of the future replace cityscapes of the present: "Critics find in Sze's work a metaphor for today's dizzying, protean urban fabric, an analogy to the cyberworld" (Carlock 2003), or "her fixation remains what is sometimes called Nature 2-the eco-techno system we inhabit and produce" (Richard 2010:256), or "Sze has a gift for invoking dimensions both prosaic and galactic" (Lehmann 2015). While they share much with her earlier work, these new pieces call into question one's understanding of the Sze type. Potentially, this new work forces audiences to expand the type to include more abstract, floating, and futuristic, and futuristic elements. Perhaps the type constricts to exclude urban design elements as indicative of the type. The point is, new tokens lead people to reflect upon the type-token relations, often with the effect of maintaining, revising, or upending the type.

Marina Abramović, the famous performance artist, offers another angle into the question of indexicalization and cultural change. A problem somewhat unique to performance art is that the artist cannot be in multiple places at once. Therefore, unlike sculptures or paintings or films, the artist must attempt to produce an exact token day after day despite always differing conditions. Even in these cases where the artist literally embodies their work of art - or rather, their art is their body - audiences will never witness the same, exact performance twice. There will always be slight changes and deviations,

\footnotetext{
${ }^{12} \mathrm{An}$ image of this piece can be found at the Tanya Bonakdar Gallery: http://www.tanyabonakdargallery.com/artists/sarah-sze/series-sculptureandinstallation_7/30. Note that images may be added to this photo album, so this URL may not link to the appropriate image at a later date if new pieces are added.
} 
intended or unintended.
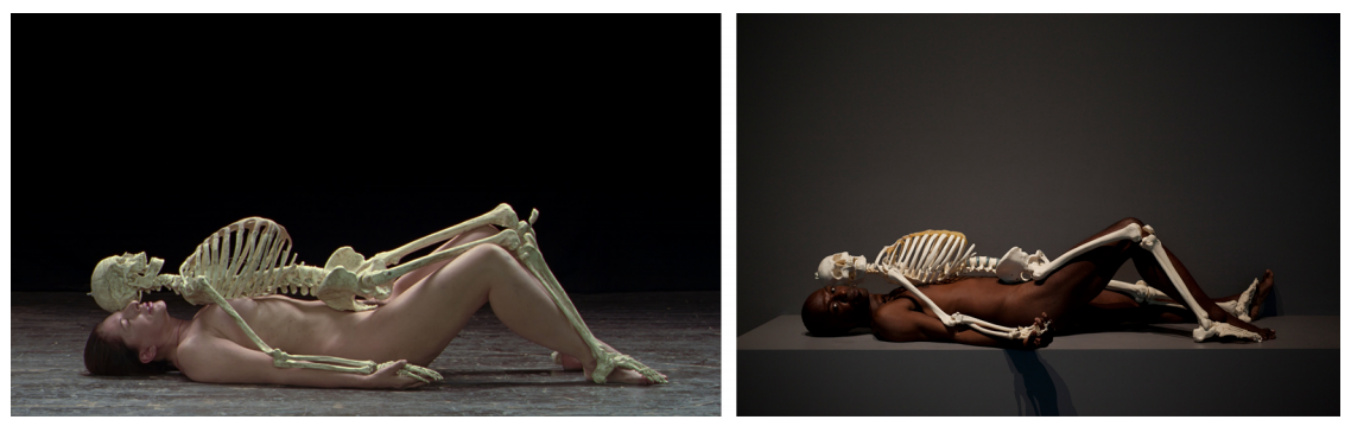

Figure 6: (Left) Marina Abramović, Nude with Skeleton, 2005. Courtesy: Marina Abramović Archives; (Right) Abramović, Marina (1946) ${ }^{\circledR}$ ARS, NY. Nude with Skeleton. Pictured: David Thomson. Installation view of the exhibition, "Marina Abramović: The Artist is Present." March 14, 2010 through May 31, 2010. The Museum of Modern Art, New York. Photo: Jonathan Muzikar ${ }^{\odot}$ The Museum of Modern Art, New York.

In the recent retrospective of her work at the Museum of Modern Art, Abramović's earlier performances were "reperformed" (Lambert-Beatty 2010) by young performance artists (see Figure 6). This is a situation in which the artists attempted to reproduce as closely as is possible a prior instantiation. Even the most faithful reproductions will always fail to be exact. Here, there are many forms of tension-reperformances must overcome the passage of time from the original installment wherein many important details may be forgotten, and of course these young artists have different bodies, with different shapes and dispositions. However, even when the organization of the reproduction removes these sources of tension-i.e., those produced by distributing tokens across time and person - cultural change is still unavoidable.

In performing The Artist is Present, one after the other attendees would silently sit across from Abramović, who would stare into their eyes (see Figure 7). Despite the simple structure of the piece, variation was rather wide. Attendees remained seated for different lengths of time. Some appeared stoic while many others cried. Some interacted with the work as intended, others violated unspoken rules (as when one museum guest disrobed and was immediately escorted away). Certainly there are recurring patterns upon which an observer could focus attention, but there are also always differences, sometimes subtle, and other times stark. All the pieces in this retrospective demonstrate that any attempt to externalize a type into a token replica will always be met with unique and unintended conditions. Cultural reproduction 


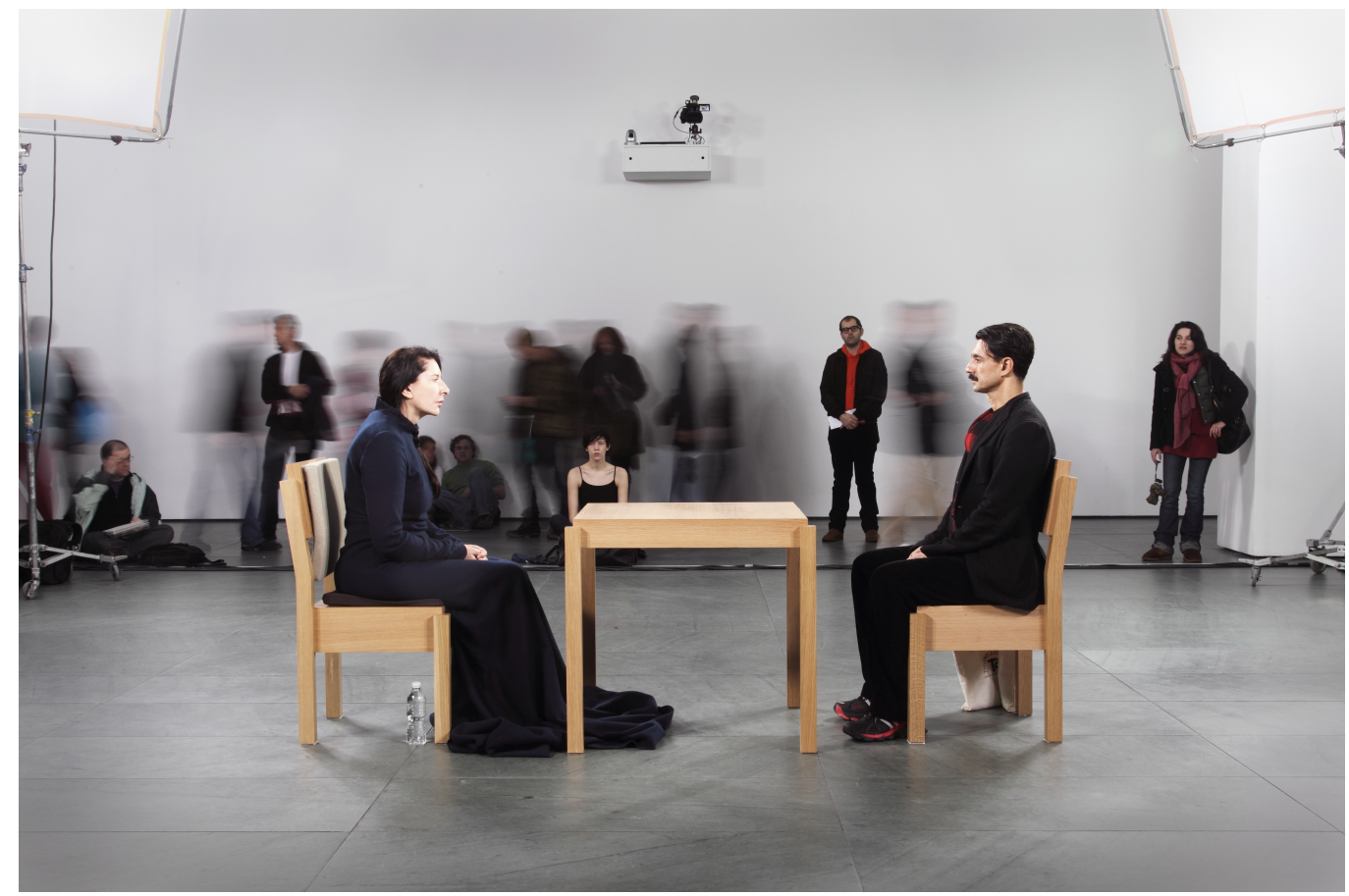

Figure 7: Marina Abramović, The Artist is Present, 2010. Courtesy: Marina Abramović Archives.

is never one-to-one.

\section{Discussion: Binding, Resonance, and Cultural Change}

Cultural objects have the appearance of stability. But, that stability takes work: binding is not simply "downloading" types. Given insights from neuroscience, we now understand cultural objects as an ongoing accomplishment through processes of indexicaling and innovating. People's binding of significance to form varies from person to person. How objects are embodied schematically leads us to rethink types as fuzzy, with variation from person to person based on their prior engagement with objects. Field dynamics and shared positions help to stabilize objects by orienting participants' attention to some qualities over others. But attention to binding makes clear how acts of maintenance are also acts of revision that occur in how people put objects to use. Importantly, innovating and indexicalizing - the processes of binding 
significance to form-occurs in the act of doing something 13 If "neurons that fire together, wire together," they fire together when people interpret objects or put them to use. As objects are used to solve problems, the bindings change for people, opening up new lines of action. The binding between types and tokens, therefore, is part of the process of cultural change.

In outlining how the binding problem relates to the study of cultural objects, this paper makes contributions to work on endogenous cultural change (Kaufman 2004; Lieberson 2000) that takes a more ecological approach (Gibson 2014). By highlighting the always tenuous binding between cultural objects as tokens and cultural objects as types, we have also highlighted the power of the passive, unacknowledged, and unconscious to produce endogenous cultural change - that is, the production of new bindings through subtle shifts in what are otherwise portrayed as "meaning-maintenance" practices (Patterson 2014:7). Furthermore, binding also plays a role in the more traditional understanding of cultural change, involving heightening emotion and awareness, conscious deliberation, and active participation in unsettled times. As an illustration, we briefly consider the relationship between the theory of resonance (McDonnell 2014; McDonnell, Bail, and Tavory 2017; Schudson 1989) and our processes of innovating and indexicalizing.

\subsection{Innovating and Resonance}

The link here between the process of "innovating" and "resonance" is especially clear. Innovating often emerges when creating or consuming novel objects - or the convolution of two or more already familiar cultural objects into something new. If resonance is "an experience emerging when affective and cognitive work provides actors with novel ways to puzzle out, or solve, practical situations," this element of novelty shares commonalities with innovating binding processes (McDonnell et al. 2017:3). As people solve problems, such as understanding a work of art that defies explanation, people scramble to make sense of it. Some may work through it, actively convolving schema, crafting form from complexity. A novel binding that makes sense of the artwork may lead to an experience of resonance ${ }^{14}$ given that it may satisfactorily fit with people's preferences and tastes. The novel bindings

\footnotetext{
${ }^{13}$ Thanks to an anonymous reviewer for pushing us to clarify this point.

${ }^{14}$ Of course, resonance is but one way an actor can experience art. Some other example experiences include apathy, disgust, joy, frustration, etc.
} 
emerging from Sze's work might lead to new paths of action. It might motivate artists to experiment along similar paths and establish new styles of art. Or it permits an art historian or curator to see previously unarticulated patterns across artworks and eras. In this sense, novel bindings set the stage for cultural change, but they often (but not always) require resonance to motivate people's commitment to the paths opened up, encourage the diffusion of such objects, and spread the object to audiences who might similarly experience resonance or to organizations that could back the object with resources.

Importantly, however, not every novel binding yields resonance. A new binding that solves one person's problem may not do so for others. Just because Sze's work could be likened to a cityscape of everyday objects, it does not mean it will resonate with people given that they may not know "why" they should care. Seeing Sze's objects, some may avoid this sense-making work by pejoratively explaining it away as "art." This is why explaining a work of art (i.e., sharing innovated bindings) might not lead to appreciation. As with much contemporary art, people without a "taste" for art after the conceptual turn may still be frustrated even when the art is explained. That said, should resonance co-occur with the establishment of an innovative binding, we would hypothesize that this binding will be better bound to memory and more likely to influence action in the future.

\subsection{Indexicalizing and Resonance}

While the process of indexicalizing is certainly put to use in cultural maintenance (Dominguez-Rubio 2014) as additional tokens affirm the type, seemingly innocuous and subtle differences across tokens can expand the boundary of what becomes included in the type. Attention to indexicalizing helps make sense of this kind of incremental change, because tokens always harbor the potential to expand the type by binding new qualities to the type. However, can such incremental and unintended changes lead to resonance?

We argue that objects sufficiently different from the common tokens of a type, but nevertheless close enough to be recognized as a token of that type, may produce resonance. ${ }^{15}$ The most imminent "problem" is, in fact, created

\footnotetext{
${ }^{15}$ There is literature on the relating concept of the "novelty-familiarity continuum" from across consumer psychology, tourism studies, and "experimental aesthetics" that scholars may wish to explore as they further refine the relationship between indexicalizing and resonance in future research (e.g., Colman, Sluckin, and Hargreaves 1981; North and
} 
by the new object-how does this fit within her oeuvre? - but requires some work to fit that object within the type; that is, it requires type maintenance or revision. In this sense, new objects to a genre may fit the genre well and not require much thinking, while others may not fit as well. In such cases people may reject the object as not a token of the type, or in other cases, revise the type to incorporate the token. Tokens can lead to resonance by forcing a type to be revised or by revealing previously unrecognized elements of the type.

\section{Conclusion}

\subsection{Summary of Argument}

In this article, we theorize the tension between meaning-making and meaning-maintenance, particularly the extent to which actors rely on one or both of these processes for instilling meaning in their phenomenological experiences. Both meaning-making and meaning-maintenance can be understood as the processes by which significance binds to material forms to either create a new cultural object (meaning-making) or to perpetuate a preexisting cultural object across space and time (meaning-maintenance). By directly theorizing the nature of these binding processes, we argued, it can be seen that processes of meaning-making and maintenance are grounded in distinct cognitive processes. Further, we showed that while individuals may be "cognitive misers" (Fiske and Taylor 1991), in the sense that they tend to maximize schematic efficiency and therefore function more as "meaning maintainers" than "meaning makers" (Patterson 2014:7), these efforts to maintain pre-existing bindings often require the creation of other new, novel bindings. A new token representation of a type, for example, often necessitates the revision of that typeeither subconsciously through the automatic recognition of family resemblances or through more effortful cultural work. In this sense, the chronicity of maintaining existing bindings often behooves the creation of new ones.

We presented this argument in three steps. First, we argued that two distinct classes of cultural objects found in previous literatures are tokens and types. The former include a single performance of Shakespeare's Julius Caesar, while the latter include "Shakespearean plays" or even "theater in

Hargreaves 1996; Lamont and Webb 2009:223). 
general." While these two are distinct, we point out the constant interplay between both cultural objects as tokens and cultural objects as types. This begs the question of how an individual can assimilate a discrete token to an existing type, and how interactions with various tokens can result in the creation of new types.

The second step involved a consideration of how the brain stores memories, and how situations invoke these memories once stored-or in Griswold's words, how individuals in a community acquire a "horizon of expectations" and how cultural objects engage these expectations. Rather than imbibing experience as a video recording, or translate experience into an amodal symbolic language, the mind encodes the recurring patterns of perceptual experience, and - through neural reuserecruits these patterns for later sensemaking. While we argued that any account of cultural objects must take seriously these cognitive regularities, it is equally true that this account must take seriously the materiality of the world. In particular, because our brains have evolved to prioritize efficiency over accuracy, and because our brains are so prone to forming associations, most humans demonstrate the common tendency to forget details and specifics, but recall very general, imprecise notions (Wood et al. 2018). This is true for actual autobiographical episodes as well as memories of tangible cultural objects. Therefore, humans offload this process into the world by inscribing culture through activities like art and text, but also because we can "visit and revisit the real-world scene, sampling it in detail at specific locations as required" (Clark 1998:29).

Taken together, this leads to our final step-which involved rejecting a one-to-one correspondence for an always-partial correspondence between public and private culture. In other words, we called for a move from crisp sets to fuzzy sets. Cultural sociologists must recognize that the mind does not assimilate an exposure to a token to its respective type by recourse to rules of necessary and sufficient conditions - as in classical categories-but rather through the logic of partial fit, as fuzzy sets. What this means is that cultural objects are always at risk of being pulled apart and appropriated in practice, and cultural change is always imminent. We find the seeds of change even in the very best attempts to replicate an artwork over time, which by generalization suggests that cultural change is likely to be the natural state of affairs. In other words, cultural stasis is likely to be a much more difficult social process than previously assumed (cf. Dominguez-Rubio 2014).

How is significance embodied in form? We've argued that significance binds to form through two processes: innovation and indexicalization. What 
consequences do these insights have for research in cultural sociology? Cultural sociologists shouldn't treat all types of meaning-making the same. Cultural objects lead dual lives as both cognitive types and material tokens. Understanding indexicalization and innovation, the processes that bind these types and tokens together, provides an account for how a cultural object emerges or otherwise remains stable over time and space. Looking closely at binding processes suggests meaning "making" and "maintenance" are not mutually exclusive. Maintenance efforts often involve subtle tweaks, alterations, and revisions that are innate to the imperfect nature of the reproduction process: cultural change is often executed in the service of cultural stability.

Finally, this paper underscores the importance of thinking of a cultural object as existing as both type(s) and token(s). Just as material instantiations of the "same" cultural object are not all the same (McDonnell 2010, 2016), cognitive types might be "shared" but are by no means identical. How people internalize cultural objects is mediated by past experience, a persons perceptual capacities, and differentially encoded in memory. Rather than ignoring this variation as noise, or only attending to the qualities shared across a group, see this inherent instability as a mechanism of change. Attending to cognitive processes of indexicalizing and innovating offers a window into the dynamic process of meaning making, maintenance, and cultural change.

\subsection{Implications for Sociological Practice}

How does understanding the binding process change sociological practice? For one, it raises questions about whether binary codes are as crisp and stable as we tend to treat them. Types are built from (often unpredictable) experience with tokens. Types are not consistent over time and space. For any one person or community, what counts as, say, "art" depends upon their history of token engagement. Patterson is right that interaction orders tend to stabilize types, but we'd argue that much of that stabilization is assumed in cultural sociology. In more rigorous fields, where definitions are poured over and debated, stabilization is more common. The same is true given perceptual primacy - visual qualities, like colors, are more codified and stable across people, whereas smell and touch are less so (Majid et al. 2018). Most of the time our intersubjective understanding of a type is assumed rather than explicitly negotiated - common problems tend to draw out this cultural work of ordering and defining types. 
To return to our discussion of cultural capital, consider Halle's Inside Culture (1993), which upended the assumption that elite taste for abstract art was widespread among the upper-class. Halle went into peoples homes to see what art people had on their wall, and while many upper-middle class urban homes had abstract art, they often indexicalized it as "decor" more than art. In addition, they had a difficult time talking about it in terms of abstraction, instead likening it to the landscape art they were familiar with. Their interpretations of abstract paintings did not align with sociological expectations, because we assumed a history of engagement with tokens and fields that would give these art owners the cultural capital to interpret this work in more sophisticated ways. Instead, they probably knew from interacting with other wealthy Americans they should value and have abstract art, but indexed these paintings more powerfully as decor. If you asked other wealthy urbanites working in the field of art, youd find very different kinds of indexicalizing and innovating. Taste for particular kinds of art depends upon ones embodied schematic mapping. One's schematic map depends on ones history with tokens of "art" built over a lifetime of engagement. The variety of tokens or the the regularity and participation across multiple fields that is may overlap more or less with others in similar positions. The degree of overlap is important, suggesting the distribution of consistency varies across fields. Solely reading meaning from objects and who owns or engages them, without attendant data on audiences interpretations, may miss important variation.

Empirically, we should treat crisp and stable types as an accomplishment, not something that should be assumed a priori. In this sense sociologists should establish the degree of "sharedness" of a cultural object. That is easier said than done. In an ideal world, wed have observed people over their long history interacting with the cultural objects we care about and have good measures of their valuations of what counts. That way we could see how much schematic overlap there might be in a field. Of course, this is an expensive prospect. Given the difficulties with just such a longitudinal approach, observing people engaged in the act of innovating and indexicalizing seems like another path. When engaging with a new object, do people innovate a type? Or indexicalize to a type? When doing so, what kinds of schematic analogies do people make by referring to other tokens?

In terms of studying the indexicalizing process as a mechanism of cultural change, this paper calls for research designs that facilitate measuring the subtle, dynamic work that goes on between actors as they attempt to give off the 
semblance of a stable type to outside audiences. Rubio (2014), for instance, used participant observation among curators and conservators to document the processes through which art objects are stabilized in museum spaces what we would refer to as token stabilization. In light of this discussion on binding, however, we would also ask: How might this interactional work between curators and conservators also be in the service (or not) of type stabilization? Does the work to stabilize artworks that require little upkeep lend itself to preserving how that particular artwork indexicalizes the type originally envisioned by the art producer? Or do attempts to stabilize "unruly" (Rubio 2014) artworks that require frequent upkeep lead to the somewhat ironic outcome where the artwork indexicalizes a new type for some audience members? For example, while replacing U-Matic cassettes with laser-discs in aging mixed-media installations gives off the semblance of token stabilization (i.e., the art object keeps functioning; see Rubio 2014:635), does this maintenance work lead to the installation indexicalizing previously unrelated typessuch as "digital technology" or "art preservation"? These are but a few of the questions for sociological research when we consider the role of binding in processes of dynamic, endogenous cultural change.

\section{References}

Anderson, Michael. 2010. "Neural Reuse: A Fundamental Organizational Principle of the Brain." Behavioral and Brain Sciences 33(4):24566.

Anderson, Michael. 2016. "Précis of After Phrenology: Neural Reuse and the Interactive Brain. Behavioral and Brain Sciences 39:e120.

Barsalou, Lawrence. 1999. "Perceptions of Perceptual Symbols." Behavioral and Brain Sciences 22(4):63760.

Berezin, Mabel. 1994. "Cultural Form and Political Meaning." American Journal of Sociology 99(5):123786.

Berger, Peter and Thomas Luckmann. 1966. The Social Construction of Reality. Anchor Books.

Biggart, Nicole and Thomas Beamish. 2003. "The Economic Sociology of Conventions." Annual Review of Sociology 29:443-64.

Bielby, William and Denise Bielby. 1994. "All Hits Are Flukes': Institutionalized Decision Making and the Rhetoric of Network Prime-Time Program Development." American Journal of Sociology 99(5):12871313.

Bloch, Maurice. 2015. In and Out of Each Other's Bodies. Routledge. 
Blumer, Herbert. 1969. "Fashion: From Class Differentiation to Collective Selection." The Sociological Quarterly 10(3):27591.

Bowden, Edward and Mark Jung-Beeman. 2003. "Aha! Insight Experience Correlates with Solution Activation in the Right Hemisphere." Psychonomic Bulletin Review 10(3):73037.

Browne, Antony and Ron Sun. 1999. "Connectionist Variable Binding." Expert Systems 16(3):189207.

Bourdieu, Pierre. 1977. Outline of a Theory of Practice. Cambridge University Press.

Bourdieu, Pierre. 1984. Distinction: A Social Critique of the Judgement of Taste. Routledge.

Bourdieu, Pierre. [1979] 1993. The Field of Cultural Production. Columbia University Press.

Carlock, Marty. 2003. "Sarah Sze's Organized Chaos." Sculpture 22(9).

Cerulo, Karen A. 2002. "Establishing a Sociology of Culture and Cognition." Pp. 1-14 in Culture in Mind: Toward a Sociology of Culture and Cognition, edited by K. A. Cerulo. Routledge.

Cisek, P. 1999. "Beyond the Computer Metaphor: Behaviour as Interaction." Journal of Consciousness Studies 6(11-12):12542.

Clancey, William. 1997. Situated Cognition. Cambridge University Press.

Clark, Andy. 1998. Being There: Putting Brain, Body, and World Together Again. The MIT Press.

Colman, Andrew M., Wladyslaw Sluckin, and David J. Hargreaves. 1981. "The Effect of Familiarity on Preferences for Surnames." British Journal of Psychology 72(3):363-69.

D'Andrade, Roy. 1987. "A Folk Model of the Mind." Pp. 11248 in Cultural Models in Language and Thought, edited by D. Holland, N. Quinn, D. Holland, and N. Quinn. Cambridge University Press.

D'Andrade, Roy. and Claudia Strauss. 1992. Human Motives and Cultural Models. Cambridge University Press.

Dominguez-Rubio, Fernando. 2014. "Preserving the Unpreservable: Docile and Unruly Objects at MoMA." Theory and Society 43(6):61745.

Edgell Becker, Penny. 1998. "Making Inclusive Communities: Congregations and the 'Problem' of Race." Social Problems 45:451-72.

Edgell Becker, Penny. 1999. Congregations in Conflict: Cultural Models of Local Religious Life. Cambridge University Press.

Emirbayer, Mustafa and Douglas W. Maynard. 2011. "Pragmatism and Ethnomethodology." Qualitative Sociology 34(1):22161. 
Feldman, Jerome. 2013. "The Neural Binding Problem(s)." Cognitive Neurodynamics $7(1): 111$.

Fillmore, Charles. 1975. "An Alternative to Checklist Theories of Meaning." Pp. 12331 in Annual Meeting of the Berkeley Linguistics Society. Berkeley, CA.

Fiske, Susan and Shelley Taylor. 1991. Social Cognition. McGraw-Hill.

Garfinkel, Harold. 1967. Studies in Ethnomethodology. Blackwell Publishers.

Gibson, James. 2014. The Ecological Approach to Visual Perception. Psychology Press.

Giddens, Anthony. 2013. New Rules of Sociological Method. John Wiley Sons.

Glenberg, Arthur. 1996. "What Is Memory For: Creating Memory in the Service of Action." Behavioral and Brain Sciences 20(1):41-50.

Goldstone, Robert. and Lawrence Barsalou. 1998. "Reuniting Perception and Conception." Cognition 65(2):23162.

Goodenough, Ward. 1970. Cultural Anthropology and Linguistics. BobbsMerrill.

Griswold, Wendy. 1986. Renaissance Revivals: City Comedy and Revenge Tragedy in the London Theater, 1576-1980. University of Chicago Press.

Griswold, Wendy. 1987a. "A Methodological Framework for the Sociology of Culture." Sociological Methodology 17:1-35.

Griswold, Wendy. 1987b. "The Fabrication of Meaning: Literary Interpretation in the United States, Great Britain, and the West Indies." American Journal of Sociology 92(5):10771117.

Griswold, Wendy. 2012. Cultures and Societies in a Changing World. Sage.

Griswold, Wendy, Gemma Mangione, and Terrence McDonnell. 2013. "Objects, Words, and Bodies in Space: Bringing Materiality into Cultural Analysis." Qualitative Sociology 36(4):43-364.

Goodenough, W.H. 1956. "Componential Analysis and the Study of Meaning." Language 32(1):195-216.

Halle, David. 1993. Inside Culture: Art and Class in the American Home. The University of Chicago Press.

Harnad, Stevan. 1990. "The Symbol Grounding Problem.' Physica D. Nonlinear Phenomena 42(1):33546.

Hebb, Donald. 1949. The Organization of Behavior: A Neuropsychological Approach. John Wiley Sons.

Heise, David. 2002. "Understanding Social Interaction with Affect Control Theory." Pp. 1740 in New Directions in Contemporary Sociological 
Theory, edited by M. Z. J Berger. Rowman Littlefield.

Heritage, John. 1987. "Ethnomethodology." Pp. 224272 in Social Theory Today, edited by A. Giddens and J. Turner. Stanford University Press.

Holland, Dorothy and Naomi Quinn. 1987. Cultural Models in Language and Thought. Cambridge University Press.

Hutchins, Edwin. 1995. Cognition in the Wild. The MIT Press.

Ignatow, Gabriel. 2007. "Theories of Embodied Knowledge: New Directions for Cultural and Cognitive Sociology?" Journal for the Theory of Social Behaviour 37(2):11535.

Jackendoff, Ray. 2002. Foundations of Language: Brain, Meaning, Grammar, Evolution. Oxford University Press.

Jordan, Jennifer. 2007. "The Heirloom Tomato as Cultural Object: Investigating Taste and Space." Sociologia Ruralis 47(1):2041.

Kaufman, Jason. 2004. "Endogenous Explanation in the Sociology of Culture." Annual Review of Sociology 30:33557.

Kenny, Stuart. 2016. "Artist Asks Strangers to Draw a Bicycle From Memory... Then 3D Renders the Results." Mpora. Retrieved March 30, 2017 (https://mpora.com/road-cycling/artist-asks-strangersdraw-bicycle-memory-3d-renders-results).

Keysers, Christian and David Perrett. 2004. "Demystifying Social Cognition: A Hebbian Perspective." Trends in Cognitive Sciences 8(11):5017.

Knorr-Cetina, Karin. 2014. "Intuitionist Theorizing." Pp.29-60 in Theorizing in Social Science, edited by R. Swedberg. Stanford University Press.

Lakoff, George. 1999. "Cognitive Models and Prototype Theory." Pp. 391421 in Concepts: Core Readings, edited by E. Margolis and S. Laurence. The MIT Press.

Lakoff, George and Mark Johnson. 1980. Metaphors We Live By. The University of Chicago Press.

Lambert-Beatty, Carrie. 2010. "Against Performance Art: Carrie LambertBeatty on the Art of Marina Abramović." Artforum International 48(9):208.

Lamont, Alexandra and Rebecca Webb. 2009. "Short- and Long-Term Musical Preferences: What Makes a Favorite Piece of Music?" Psychology of Music 38(2):222-41.

Latour, Bruno, Pablo Jensen, Tommaso Venturini, Sebastian Grauwin, and Dominique Boullier. 2012. "The Whole Is Always Smaller than Its Parts' - a Digital Test of Gabriel Tardes' Monads." The British Journal of Sociology 63(4):590615. 
Lehmann, Claire. 2015. "Sarah Sze: Tonya Bonakdar Gallery." Artforum International. Retrieved May 10, 2018 (https://www.artforum. com/picks/sarah-sze-54906).

Lena, Jennifer. 2012. Banding Together: How Communities Create Genres in Popular Music. Princeton University Press.

Lieberson, Stanley. 2000. A Matter of Taste: How Names, Fashions, and Culture Change. Yale University Press.

Lizardo, Omar. 2016. "Cultural Symbols and Cultural Power." Qualitative Sociology 39(2):199204.

Lizardo, Omar. 2017. "Improving Cultural Analysis: Considering Personal Culture in its Declarative and Nondeclarative Modes." American Sociological Review 82(1):88-115.

Lizardo, Omar and Michael Strand. 2010. "Skills, Toolkits, Contexts and Institutions: Clarifying the Relationship between Different Approaches to Cognition in Cultural Sociology." Poetics 38(2):20528.

Majid, Asifa, et al. 2018. "Differential Coding of Perception in the World's Languages." Proceedings of the National Academy of Sciences 115(45):1136911376.

Martin, John. 2010. "Life's a Beach but You're an Ant, and Other Unwelcome News for the Sociology of Culture." Poetics 38(2):22944.

Martin, John. 2011. The Explanation of Social Action. Oxford University Press.

McDonnell, Terence. 2010. "Cultural Objects as Objects: Materiality, Urban Space, and the Interpretation of AIDS Campaigns in Accra, Ghana." American Journal of Sociology 115(6):1800-1852.

McDonnell, Terence. 2016. Best Laid Plans: Cultural Entropy and the Unraveling of AIDS Media Campaigns. University of Chicago Press.

McDonnell, Terence, Chris Bail, and Iddo Tavory. 2017. "A Theory of Resonance." Sociological Theory 35(1):1-14.

Menary, Richard. 2010. The Extended Mind. The MIT Press.

North, Adrian C. and David J. Hargreaves. 1996. "The Effects of Music on Responses to a Dining Area." Journal of Environmental Psychology 16(1):55-64.

Olivier, Laurence. 1948. Hamlet. Two Cities Films.

Parsons, Talcott. 1964. Social Structure and Personality. The Free Press.

Parsons, Talcott, Edward Shils, and Neil Smelser. 1965. Toward a General Theory of Action. Transaction Publishers. 
Patterson, Orlando. 2014. "Making Sense of Culture." Annual Review of Sociology 40:1-30.

Peirce, Charles. 1992. The Essential Peirce: Selected Philosophical Writings, Volume 1. Edited by N. Houser and C. Kloesel. Indiana University Press.

Quinn, Naomi. 2011. "The History of the Cultural Models School Reconsidered: A Paradigm Shift in Cognitive Anthropology." Pp. 3046 in A Companion to Cognitive Anthropology, edited by D. Kronenfeld, G. Bennardo, V. de Munck, and M. Fischer. Blackwell Publishing.

Reay, Mike. 2010. "Knowledge Distribution, Embodiment, and Insulation." Sociological Theory 28(1):91-107.

Richard, Frances. 2005. "Sarah Sze: Marianne Boesky Gallery." Artforum International 44(1):303.

Richard, Frances 2010. "Sarah Sze: Tanya Bonakdar Gallery." Artforum International 49(4):255-56.

Rosch, Eleanor. 1999. "Reclaiming Concepts." Journal of Consciousness Studies 6(11-12):6177.

Salomon, Gavriel. 1997. Distributed Cognitions. Cambridge University Press.

Schudson, Michael. 1989. "How Culture Works." Theory and Society 18(2):15380.

Schwabsky, Barry. "SARAH SZE." Artforum International 38(2):143.

Scott, Andrea. 2012. "A Million Little Pieces." The New Yorker. Retrieved May 10, 2018 (https://www.newyorker.com/magazine/2012/05/14/amillion-little-pieces).

Searle, John. 2015. Seeing Things as They Are: A Theory of Perception. Oxford University Press.

Shore, Bradd. 1996. Culture in Mind: Cognition, Culture, and the Problem of Meaning. Oxford University Press.

Slater, Don. 2002. "Making Things Real Ethics and Order on the Internet." Theory, Culture \& Society 19(5-6):22745.

Stoltz, Dustin and Marshall Taylor. 2017. "Paying with Change: The Purposeful Enunciation of Material Culture." Poetics 64:26-39.

Swidler, Ann. 2001. Talk of Love: How Culture Matters. University of Chicago Press.

Thagard, Paul. 2010. "How Brains Make Mental Models." Pp. 44761 in Model-Based Reasoning in Science and Technology, edited by L. Magnani, W. Carnielli, and C. Pizzi. Springer. 
Thagard, Paul and Terrence Stewart. 2011. "The AHA! Experience: Creativity through Emergent Binding in Neural Networks." Cognitive Science 35(1):133.

Tulving, Endel. 2002. "Episodic Memory: From Mind to Brain." Annual Review of Psychology 53:125.

Tversky, Amos and Daniel Kahneman. 1973. "Availability: A Heuristic for Judging Frequency and Probability." Cognitive Psychology 5(2):207-32.

Vaisey, Stephen and Omar Lizardo. 2016. "Cultural Fragmentation or Acquired Dispositions? A New Approach to Accounting for Patterns of Cultural Change." Socius 2:1-15.

Van der Velde, Frank and Marc de Kamps. 2006. "Neural Blackboard Architectures of Combinatorial Structures in Cognition." Behavioral and Brain Sciences 29:37-108.

Vatansever, Deniz, David Menon, and Emmanuel Stamatakis. 2017. "Default Mode Contributions to Automated Information Processing." Proceedings of the National Academy of Sciences 114(48):128216

Whitford, Josh. 2002. "Pragmatism and the Untenable Dualism of Means and Ends." Theory and Society 31(3):325-63.

Whitney, David. 2009. "Neuroscience: Toward Unbinding the Binding Problem." Current Biology 19(6):R251-53.

Wilson, Margaret. 2002. "Six Views of Embodied Cognition." Psychonomic Bulletin Review 9(4):62536.

Wood, Michael Lee, Dustin S. Stoltz, Justin Van Ness, and Marshall A. Taylor. 2018. "Schemas and Frames." Sociological Theory 36(3):24461.

Zimmer, Hubert, Axel Mecklinger, and Ulman Lindenberger. 2006. Handbook of Binding and Memory. Oxford University Press. 